\section{Personale Effekte der Rekrutierung von Mädchen in technische Lehrberufe}

\section{Die Ausgangslage - Situation auf dem Arbeitsmarkt}

Viele europäische Länder leiden unter Rekordarbeitslosigkeit, gleichzeitig beklagen zahlreiche Unternehmen die schlechte Qualität von BewerberInnen (Der Standard 2005). Manche Firmen versuchen schon aktuell Nachwuchs für die Zeit des Arbeitskräftemangels, der ab 2010 bzw. 2015 für Österreich prognostiziert wird, an ihr Unternehmen zu binden (Innreiter-Moser/Littringer/Stummer 2006, 83).

Eine Strategie, diese Situation zu verändern, ist es zu versuchen, für atypische BewerberInnen - in technischen Berufen sind das Frauen oder junge Mädchen - attraktiv zu werden. In Österreich wählen im Bereich der betrieblichen Ausbildungsberufe Jugendliche ihre Berufe sehr traditionell geschlechtersegmentiert. So begannen im Jahr 2003 ca. 50\% der Mädchen eine Ausbildung als Verkäuferin, Friseurin oder Sekretärin, technische Berufe kamen unter den 10 meistgewählten Lehrberufen bei jungen Frauen nicht vor. 10\% der Jungen lassen sich zu KFZ-Technikern ausbilden, auch die weiteren, am häufigsten gewählten Arbeitsgebiete liegen mit Ausnahme von Koch/Kellner und Verkäufer eher im technisch-handwerklichen Bereich (MUT 2005). In der BRD zeigt sich eine vergleichbare geschlechtersegmentierte Berufswahl (Granato 2004, 10).

Gerade in der Altersklasse, in der die Entscheidung über eine betriebliche Ausbildung (bis zum 15. Lebensjahr) getroffen wird, scheint die Familie den wesentlichen Einflussfaktor auf die Berufswahl ihrer Kinder darzustellen (Schmid 2002, 7). Die Selbstreferenzrahmen von Jugendlichen werden laut Literatur meist von den Eltern geschlechterstereotyp geprägt(z.B.Parsons/Adler/Meece 1984,36f), sodass etwa Mädchen sowohl von Männern als auch von Frauen im Schnitt bei technischen Berufen schlechter bewertet werden (Giurleo 1997, 90).

Diese Phänomene im Arbeitsleben aufzubrechen, erfordert von betrieblicher Seite, die Personalpolitik verstärkt an Diversity-Ansätzen auszurichten.

\section{Diversity und Gender}

Im deutschsprachigen Raum sind die Forschungen zu Diversity jung, die Beiträge zur Bedeutung der multikulturellen Organisation - d.h. das organisierte und integrierte Zusammenarbeiten von Menschen mit Unterschieden - nehmen in der wissenschaftlichen Diskussion zu, wenn sie auch sehr kontroversiell ausfallen (z.B. Gebert 2004; Sackmann/Bissels/ Bissels 2002).

Der Begriff „Diversity“ in Organisationen umfasst ein breites Spektrum möglicher Vielfalt, die durch vielerlei Unterschiede zwischen den Menschen entsteht. Die angesprochenen Unterschiede werden in Diversity-Ansätzen nicht nur als Trennendes sondern auch als Verbindendes, im Sinne von Synergien und Facettenreichtum, gesehen (Aretz/Hansen 2003, 10).

Die wissenschaftliche Differenzierung möglicher Diversity-Merkmale hat unterschiedliche Systematiken hervorgebracht, in denen nach primären und sekundären Dimensionen (Loden/Rosener 1991,21) wie Alter, Geschlecht, Rasse, sexuelle Orientierung etc. (primär) und Bildungshintergrund, Herkunft, Einkommen, Berufserfahrung u.a.m. (sekundär) unterschieden wird. Auch die unterschiedlichen sozialen Ebenen, etwa Faktoren der Individualebene, wie Identität, Stereotype, Persönlichkeit, der Gruppen- und Intergruppenebene und Faktoren im organisationalen Kontext, z.B. Organisationskultur, strukturelle/formale Integration, informale Integration, werden differenziert (Cox 1996). Schließlich schlagen Jackson/Ruderman (1996) vor, zwischen demografischer Diversität (Geschlecht, ethnische Zughörigkeit, Alter etc.), psychologischer Diversität (Werte, Einstellungen und Wissen) und organisationaler Diversität (etwa Dienstalter, hierarchische Stellung, Art der Beschäftigung) zu unterscheiden. Die genannten Typologisierungen ergänzen einander und/oder überlappen sich teilweise.

Auf der Haltungs- und Verständnisebene kann Diversity nach Thomas/Ely (1996) in drei Ansätzen gefasst werden:

1. Fairness- und Diskriminierungsansatz: Hierbei geht es unabhängig von Unterschieden um eine faire Behandlung aller MitarbeiterInnen und damit um Verhinderung von Diskriminierung. Das heißt, der Arbeitsalltag wird so gestaltet, als gäbe es keine Vielfalt (Aretz/Hansen 2003, 16)

2. Marktzutritts- und Legitimitätsansatz: Durch Diversity soll der Zutritt zu diversen KundInnen gefunden werden. In der Belegschaft soll sich auch der potenzielle Markt spiegeln. Aretz/Hansen $(2003,17)$ kritisieren hierbei, dass Vielfalt nicht wirklich akzeptiert und integriert, sondern funktionalisiert wird. Stereotypisierungen sind damit Tür und Tor geöffnet.

3. Lern- undEffektivitätsansatz: Hier wird Diversity ganzheitlich - im Sinne von, dass jedes Belegschaftsmitglied seine individuelle Persönlichkeit nutzbringend für sich und die Organisation einbringen kann - verstanden, was zu hohen 
Anforderungen an die Organisationsmitglieder führt (Aretz/Hansen 2003, 17).

Abhängig vom Zugang zum Themenkomplex „Diversity” ergeben sich für die Personalarbeit in Organisationen unterschiedliche Anforderungen. Die Motive können von Marktzwängen (z.B. der monokulturelle Arbeitsmarkt liefert nicht genug „beste” Arbeitskräfte) bis zum regelrechten Suchen nach Innovation durch Diversity reichen.

Während homogene Gruppen effizienter und schneller arbeiten, scheinen heterogene Gruppen innovativer und kreativer zu sein, was aber nur zum Tragen kommt, wenn ,... die vielfältig zusammengesetzten Gruppen entsprechend trainiert werden" (Krell 2002, 113). Das bedeutet, erst das „richtige” Managen von Diversity führt auch zu Wettbewerbsvorteilen (Krell 2002, 107 f.). Eine besondere Rolle spielen dabei Führungskräfte auf allen Ebenen (Tondorf/Krell 1999).

Studien unterscheiden weiterhin zwischen sichtbaren und weniger sichtbaren Merkmalen von Teammitgliedern und verweisen auf deren Effekte im Organisationsalltag: So zeigen demografische (sichtbare) Merkmale eher affektive Konflikte, die zu negativen Auswirkungen auf Gruppenleistungen führen, und aufgabenbezogene Merkmale eher inhaltliche Konflikte, die als produktiv gelten (Sackmann/Bissels/Bissels 2002, 46).

Eine der sichtbaren demografischen Merkmalsausprägungen in Arbeitsgruppen stellt „Geschlecht” dar. Entsprechend traditioneller geschlechtsspezifischer Bilder und Rollen ist der Arbeitsmarkt in Österreich nach wie vor stark zwischen Männern und Frauen segmentiert, so auch in der Automobilindustrie, in der die nachfolgende Untersuchung durchgeführt wurde.

\section{3 „Just 4 Girls“ - ein innovatives Projekt der Magna Steyr}

Die Automobilindustrie ist traditionell „Männer-dominiert". Die Arbeitsanforderungen sind komplexer geworden, so dass neue Berufe erlernt werden müssen (z.B. Mechatronik). Zusätzlich wollen einige Firmen den Angebotsmarkt an potenziellen Auszubildenden - etwa junge Frauen - ausweiten, so auch die Firma in unserer Fallstudie.

Magna Steyr ist ein international tätiges Unternehmen in der Automobilbranche mit den Schwerpunkten Fahrzeugentwicklung und -bau. Magna International beschäftigt ca. 82.000 MitarbeiterInnen (Magna 2005), Magna Steyr als Tochtergesellschaft mit zwei Hauptwerken in der Nähe von Graz und weiteren 38 Standorten ca. 11.000 (Magna Steyr 2005). In Graz hat die Firma im technischen Bereich folgende Lehrberufe: Elektrobetriebstechnik, Karosseriebautechnik, KFZ-Technik/Elektrik, Maschinenbautechnik, Mechatronik und Zerspanungstechnik Die Ausbildungen dauern zwischen dreieinhalb und vier Jahren. Der Mädchenanteil ist im Bereich Karosseriebautechnik über-, bei Mechatronik und Maschinenbautechnik unterdurchschnittlich.

Magna Steyr nahm im Jahr 1999/2000 am österreichischen Modellprojekt zur Förderung von Chancengleichheit „Equality Management - Optimale Ressourcennutzung durch eine chancengerechte Personal- und Unternehmensführung" teil. Die Hauptkonzentration dabei galt dem Handlungsfeld „Recruiting“. Die durchgeführten Aktivitäten fokussierten die Erhöhung des Frauenanteils im technischen Bereich, insbesondere die Steigerung des Mädchenanteils in den Lehrberufen. Mit dem Programm, ,Just 4 Girls“" sollte das verändert werden. Folgende Schritte wurden gesetzt:

- Zusammenarbeit mit den regionalen Schulbehörden

- Workshop mit BerufsorientierungslehrerInnen

- Elternabende in den Schulen

- Schnuppertage für Mädchen

Insgesamt wurde ein Informationspaket präsentiert, das für Mädchen ansprechend war und Magna Steyr als zukünftigen Arbeitsplatz attraktiv machte (,Auto ist nicht nur Kraft-Männer-Öl, sondern auch Feinfühligkeit und Ästhetik"). Neben dem direkten Arbeitsplatz wurden auch die Arbeitsprozesse und ihre Verbindungen, von der Konzeption eines Tankdeckels bis zum Crashtest des fertigen Fahrzeugs, vorgeführt. Um Zusammenhänge im Unternehmen zu verdeutlichen, wurden damit die Gesamtheit und die Bedeutung der Einzelteile für das Endprodukt dargeboten.

Die Erwartungen wurden übererfüllt: Doppelt so viele LehrerInnen wie erwartet besuchten den Workshop und die Mädchenanzahl erreichte das Vierfache der Planzahlen.

Das besondere an ,Just 4 Girls“ war der Versuch, die Mädchen umfassend anzusprechen. Beispielsweise waren an den einzelnen Schnupperstationen bewusst ausschließlich junge Frauen (Mädchen aus dem zweiten/dritten Lehrjahr) als Demonstratorinnen abgestellt, um möglichst Kommunikationsbarrieren zu vermeiden

Das Programm wurde einmalig durchgeführt, wobei sich die Firma bei politischen Projekten wie "Girl's Day“ auch weiterhin beteiligt. Wie Tabelle 1 zeigt, führte die Initiative zwar zu einem kurzfristigen Erfolg, allerdings mit nachlassender Wirkung in den Folgejahren: Waren im Jahr 2002 32\% der mit der Ausbildung beginnenden Jugendlichen Mädchen, so sank der Anteil auf 19,6\% im Jahr 2003 und auf 
16\% im Jahr 2004.

Nichtsdestoweniger gab es eine Änderung der Geschlechterzusammensetzung, ursprünglich nur im Ausbildungszentrum und in den relevanten AuBenstationen, nach der Ausbildungszeit ebenfalls in den Fachabteilungen. Bei einer Veränderung der Geschlechterverhältnisse von ca. 5-7\% mehr Mädchen auf einen Anteil von 32\% bzw. in den Folgejahren $19 \%$ und 16\% an allen Auszubildenden steht zu erwarten, dass die Ausbildung bzw. Beschäftigung von Frauen auch im Produktionsbereich verstärkt zum Thema werden wird

\section{Tabelle 1: Lehrlinge im technischen Bereich der Magna Steyr.}

paraphrasiert und verdichtet. Ziel der Methode war es, aus dem analysierten Fall Hypothesen zu generieren.

\section{$5 \quad$ Hypothesen und Effekte}

\section{Hypothesen}

Die Auswertung der Interviews ergibt folgende Hypothesen, die auch im Sinne von Empfehlungen bei ähnlichen Programmen interpretiert werden können:

\section{Hypothese 1: Vielfalt an sich ist kein Wert im Produktionsbereich}

Die in der Literatur geäußerten möglichen Vorteile von Diversity werden in Produktionsbetrieben im Normalfall nicht gewünscht. Ziel ist

\begin{tabular}{|l|c|c|c|}
\hline Jahr & $\begin{array}{l}\text { Lehrlinge } \\
\text { gesamt }\end{array}$ & $\begin{array}{l}\text { Prozent der } \\
\text { aufgenommenn } \\
\text { Lehrlingeweib- } \\
\text { lich }\end{array}$ & $\begin{array}{l}\text { Prozent der Bewer- } \\
\text { bungen von Mäd- } \\
\text { chen }\end{array}$ \\
\hline 2001 & 44 & $6,82 \%$ & $7,90 \%$ \\
\hline 2002 & 50 & $32,00 \%$ & $16,03 \%$ \\
\hline 2003 & 51 & $19,61 \%$ & $8,98 \%$ \\
\hline 2004 & 50 & $16,00 \%$ & $10,09 \%$ \\
\hline
\end{tabular}

Effizienz, die eher mit homogenen Arbeitsgruppen als mit heterogenen gewährleistet ist. Im Hinblick aufKoordination und Konfliktmanagement wird der Aufwand als zu hoch angesehen (Gebert2004,425; Sackmann/Bissels/ Bissels 2002, 49).

„Ich glaube nicht, dass Vielfalt an sich wirklich erstrebenswert ist. ... ich glaube was wichtiger ist, ist eine Haltung der Offenheit gegenüber ver-

Quelle: internes Dokument der Personalentwicklung der Magna Steyr.

\section{Methodologie und Forschungsdesign}

Im Rahmen der Abschlussevaluierungen des genannten E-Quality-Projekts interviewten die AutorInnen 2003 (a) den Leiter der Personalentwicklung, der auch für die Lehrlingsausbildung zuständig ist und (b) sechs weibliche Auszubildende der Magna Steyr. Des Weiteren wurden (c) zwei KontaktlehrerInnen in Graz und Umgebung befragt. Fokus der Forschung im Jahr 2003 waren Gründe und Hemmnisse für die atypische Berufswahl der jungen Mädchen.

Im Juli 2005 fanden Folgeinterviews statt. Es wurden (a) wieder der Leiter der Personalentwicklung und (b) eines der Mädchen, das wir schon 2003 befragt hatten, aber (c) diesmal auch vier Ausbildungsmeister und (d) zwei männliche Auszubildende im zweiten Lehrjahr interviewt. Fokus waren die internen Prozesse und Bilder. Die Fragenblöcke umfassten mögliche Änderungen durch die verstärkte Hereinnahme von Mädchen in technische Lehrberufe und Erfahrungen der unmittelbar Betroffenen.

Alle Interviews wurden problemzentriert durchgeführt, aufgezeichnet und anschließend transkribiert, schiedenen Gruppen.“ (I1,13,4)

....da sollte ein Betrieb meines Erachtens das Prinzip haben ,der Beste bekommt den Job ganz egal welche Herkunft er hat, ob das männlich oder weiblich ist. ..., dann hast du automatisch Vielfalt herinnen, mit der du dich vielleicht auseinandersetzen musst ... ich sage, die Qualifikation zählt ...dann kann man auch wirklich etwas tun, wenn der Gesamtnutzen insgesamt erkennbar ist." (I1,13,4 - 14,3)

Ziel ist eine Homogenisierung - Begleitmaßnahmen für unterschiedliche Zielgruppen werden gefördert, aber mit dem Ziel, Effizienz durch Homogenität zu schaffen. Offenheit ist die Methode, Unterschiedlichkeit in den Eingangsbedingungen abzugleichen. Damit wird nach Krell $(2002,108)$ trotz bestehender Vielfalt sehr beständig und erfolgreich an so genannten ,homogenen Idealen” festgehalten. Bei Hypothese 1 zeigt sich zwischen den befragten Gruppen kein erkennbarer Unterschied.

\section{Hypothese 2: Mädchen in technischen Berufen befinden sich in einer, ,Token- Situation"}

Mädchen stellen in allen technischen Berufsrichtungen bei Magna Steyr eine Minderheit dar. Das gibt ihnen einen eigenen Status. Sie sind aufgrund 
ihres Geschlechtes besonders ,sichtbar", was einerseits als positiv anspornend erlebt werden kann und andererseits negativ hohen Druck bedeuten kann (Kanter 1977, 210f).

„Weil wir Frauen müssen uns erst einmal beweisen am Anfang“. (I2, 2, 7), ,... und auffallen tust du da gleich einmal"“ $(\mathrm{I} 2,4,4)$.'

Andererseits greifen die Medien Mädchen in technischen Lehrberufen besonders heraus.

„Wenn ein Betrieb drei Mädchen aufnimmt, dann steht er in der Zeitung. Nimmt er 50 Buben auf, interessiert es keinen Menschen“ (I3, 6, 7).

Hierbei treten unterschiedliche Wahrnehmungen auf, sehen die Ausbildner die Mädchen und diese auch sich selbst eindeutig in einer Token-Situation (Kanter 1977, 210f), so deuten die Jungen dies nur für die Anfangsphase so.

Hypothese 3: Personalmarketing für Mädchen in technische Berufe muss wiederholt und auf unterschiedlichen Ebenen betrieben werden.

Personalpolitische Änderungen beim Ansprechen von unterschiedlichen Gruppen für die Lehrlingsausbildung benötigt ein differenziertes Personalmarketing (Innreiter-Moser/Stummer 2003, 7f). Geschlechtsspezifische Berufsbilder sind in unserer Gesellschaft tief verwurzelt und können auch nicht mit Einmalmaßnahmen wie „Just 4 Girls“ aufgebrochen werden.

Um hier dauerhafte Wirksamkeit zu erreichen, müssen nicht nur Mädchen zumindest derzeit für technische Berufe laufend beworben werden, sondern auch die dominant prägenden Institutionen (Schule, Familie u.a.)

„... das gehört viel mehr gezeigt, dann trauen sie [die Mädchen] sich auch mehr" (I3, 8, 12)

„, Das heißt also, wenn man nicht sehr vehement am Markt auftritt, dann kommen die Mädchen trotzdem nicht, auch wenn man vielleicht das Image hat einer mädchenfreundlichen Lehrwerkstätte. ... ständig zu informieren, und ständig zu beknien und sehr darum zu ringen, dass Mädchen bereit sind in die technische Lehrausbildung zu kommen." (I1, 2, 2).

„Es geht einfach darum, dass das im Kopf der Eltern und im Kopf der Lehrer ist.” (I3, 15, 7).

„...es müsste generell ein bildungspolitisches Thema werden ...ich versuche Schritt für Schritt Interessensträger und Körperschaften dafür zu gewinnen, da nachhaltiger ... und da konkreter und systematischer zu sein ....in der Volksschule anfangen und Kindergarten möglicherweise, und die sehr viel mit dem zu tun haben, dass sich die Berufsorientierung auch mit Eltern auseinandersetzt und nicht nur mit den Kindern selbst.“ (I1, 11, 1)
„..., wir müssen, wenn wir das wirklich weiterhin betreiben und weiterhin wollen ..., dann muss man permanent Werbung machen. Es geht nicht anders." $(\mathrm{I} 4,4,5)$.

Bei Hypothese 3 herrscht weitgehend Einigkeit zwischen den InterviewpartnerInnen, es werden häufig das Elternhaus und die Schulen, insbesondere von den Mädchen auch die BerufsorientierungslehrerInnen kritisiert.

\section{Beabsichtigte Effekte}

„Just 4 Girls“ wirkte sich zahlenmäßig positiv aus, d.h. die Zielgröße wurde erreicht, dieAuszubildenden konnten aus einer wesentlich größeren Bewerberanzahl ausgewählt werden und somit weiterhin eine sehr hohe Qualität aufrechterhalten werden.

Wie es scheint, passen sich die Mädchen größtenteils an das herrschende Berufsbild an, und es wird berichtet, dass die Jungen ihren Sprachgebrauch tendenziell mäßigen. Wie es ein teilnehmendes Mädchen ausdrückte: ,Alleine dadurch, dass ich so lange mit den Männern arbeite, bin ich schon auch anders geworden. Also man passt sich irgendwie schon an“ $(I 2,7,4)$. Auch die befragten Jungen kommentierten es wie folgt: „Also sie passt sich nachher schon quasi an, sie schauen sich so quasi die Burschen an..." (I6, 5,14).

Insgesamt ist der Frauenanteil an den Beschäftigten bei Magna Steyr gestiegen. Das hängt zwar nur in kleinem Maße mit den Auszubildenden zusammen. Den Anteil der Frauen an allen Beschäftigten weiter zu erhöhen, ist aber durchaus ein langfristiges Ziel der Firma.

\section{Unbeabsichtigte Wirkungen}

In manchen Lehrberufen waren nach Meinung der Ausbildungsmeister zu viele Mädchen, die als Gruppe schwierig zu leiten waren. Sie hatten keine bis wenig Erfahrung mit der Dynamik der Mädchen untereinander und der Einbringung von „Privatempfinden“. Von männlichen Gruppen war ihnen dies nicht bekannt. Mädchen wurde in der Befragung nachgesagt, interne Konkurrenz anders auszutragen und die Erhöhung über einen „gesunden“ Anteil scheint negativ zu wirken. ,... weil wenn mehr Mädchen sind, da habe ich zu viel gestritten“ (I2, 2, 3).’ „Aber man muss immer damit rechnen, wenn mehrere [Frauen] beieinander sind, dass da mehr gestritten wird" (I2, 11, 3). „Nein, Konflikte, interessanterweise die Mädchen untereinander. Also, nicht mit den Ausbildnern, nicht mit den Burschen, untereinander. Also da hapert es am meisten“ (I3, 9, 4).

Betriebliche Anpassungen gibt es laufend bei der Kleidung: Die Anfangsprobleme, dass Schuhe, Kleidung undArbeitshandschuhe für Mädchengrößen 
nicht vorhanden waren, sind gelöst, von kleineren Schwierigkeiten wird aber noch weiter berichtet.

\section{Resümee}

Der vorliegende Beitrag ist eine Untersuchung über personale Effekte des Einbindens von Mädchen in technische bisher monokulturell männlich besetzte Lehrberufe. Bei oberflächlichem Hinsehen offenbaren sich kaum Unterschiede zwischen männlichen und weiblichen Auszubildenden, Abweichungen tauchen lediglich peripher auf. Motiv dafür scheint ein Firmencommitment im Sinne des Fairness- und Diskriminierungsansatzes zu sein. Magna Steyr setzte mit „Just 4 Girls“ einmalig einen Schwerpunkt und signalisierte damit, dass junge Mädchen als Auszubildende nachgefragt werden und auch dieselben Chancen haben wie Jungen.

Besonderheiten im Personalmarketing werden akzeptiert, ansonsten betonen alle Befragten, eine absolute Gleichbehandlung der Auszubildenden zu verfolgen. Die weiblichen Auszubildenden scheinen sich den bestehenden Berufsnormen entsprechend anzupassen, auffallend zeigte sich auch eine äußerst männliche Sprache aller InterviewpartnerInnen. Homogenität fördert Effizienzund ist von den Ausbildungsmeistern gewünscht. Verschiedene Äußerungen in den Interviews zeigen dennoch, dass auch bei Magna Steyr die Auszubildenden ein unterschiedliches Geschlecht haben, Obwohl alle Befragen beteuern, sie absolut gleich zu behandeln. Eine dauerhafte Wirksamkeit der untersuchten personalpolitischen Maßnahmen ist zwar sichtbar, wenn auch schwach ausgeprägt. Eine Ausstrahlung auf andere Betriebe ist trotz breiter politischer Propagierung von „,Frauen in technische Berufe" nicht erkennbar. Um dies zu erreichen, müsste voraussichtlich an gesellschaftlich dominanten Institutionen angesetzt werden. Studien aus der BRD berichten von ähnlichen Vorgehensweisen und auch vergleichbaren Schwierigkeiten (vgl. BIBB 2003; Granato 2004; Ernst u.a. 2006).

\section{LITERATUR}

Aretz, Hans-Jürgen, Katrin Hansen (2003): Erfolgreiches Management von Diversity. Die multikulturelle Organisation als Strategie zur Verbesserung einer nachhaltigen Wettbewerbsfähigkeit; in: ZfP, 17 (1), 9-36

BIBB (2003): Auszeichnung für Innovative Berufsbildung. Hermann-Schmidt-Preis 2003; in: BWPspezial, 8, 1-12

Cox, Taylor H., Stacy Blake (1991): Managing cultural diversity: implications for organizational competitiveness; in: Academy of Management Executive, 5 (3), 45-56

Der Standard (2005): Qualitätsprobleme waren vorher- sehbar. Georg Toifl, Bundesobmann Gewerbe und Handwerk der Wirtschaftskammer im Interview, 1. Februar

Ernst, Helmut, Liane Mähler, Ralph Schott, Ute Vatter (2006): Unterstützungssysteme für Betriebe. Strategien zur Integration von Jugendlichen mit besonderem Förderbedarf. BIBB, Bonn

Gebert, Diether (2004): Durch diversity zu mehr Teaminnovativität? Ein vorläufiges Resümee der empirischen Forschung sowie Konsequenzen für das diversity Management; in: DBW 64 (1), 412-430

Giurleo, Susan (1997): Persisters and Career Changers in Technical Careers. Are There Gender Differences? in: Helen S. Farmer andAssociates (1997): Diversity \& Women's Career Development. From Adolescence to Adult. Thousand Oaks u.a., 81-94

Granato, Mona (2004): Potenziale junger Frauen nutzen; in: ibv, 22, 7-18

Innreiter-Moser, Caecilia, Marianne Littringer, Harald Stummer(2006): Führung in Teilzeit-Geschlechtsspezifische und organisationale Bedingungen; in: R. Bendl (Hg): Betriebswirtschaftslehre und Frauen- und Geschlechterforschung - Empirische Ergebnisse zur Standortbestimmung der Betriebswirtschaftslehre. Frankfurt/Main, 81-101

Innreiter-Moser, Caecilia, Harald Stummer (2003): Achieving Diversity? Paper presented at the $19^{\text {th }}$ EGOS conference. Copenhagen, July 3-5

Jackson, Susan E., Marian N. Ruderman (Hg.) (1996): Diversity in Workteams. Research Paradigms for a Changing Workplace. Washington, D.C

Kanter, Rosabeth M. (1977): Men and Women of the Corporation. New York

Krell, Gertraude (2002): Diversity Management. Optionen für (mehr) Frauen in Führungspositionen? in: Sibylle Peters, Norbert Bensel (Hg.): Frauen und Männer im Management. Diversity in Diskurs und Praxis. Wiesbaden, 105-120

Loden, Marilyn, Judy B. Rosener (1991): Workforce America!: Managing Employee Diversity as a Vital Resource. Homewood

Magna (2005): Homepage der Magna Firmengruppe. URL: http://www.magna.com. dl: 3.09.2005

Magna Steyr (2005): Homepage der Magna Steyr. URL: www.magnasteyr.com. dl: 3.09.2005

MUT (2005): Mädchen und Technik. URL: http://www. mut.co.at. dl: 1.09.2005

Parsons, Jaquelynne E., Terry Adler, Judith L. Meece (1984): Sex differences in achievement: A test of alternate theories; in: Journal of Personality \& Social Psychology 46 (1), 26-43

Sackmann, Sonja, Sandra Bissels, Sandra, Thomas Bissels (2002): Kulturelle Vielfalt in Organisationen: Ansätze zum Umgang mit einem vernachlässigten Thema der Organisationswissenschaften; in: DBW 62 (1), 43-58

Schmid, K. (2002). Familie und Berufswahl. Paper presented at the ÖIF-Symposium „Zwischen Identität 
und Provokation. Das Spannungsfeld Jugendliche- Erwachsenwerden Familie“. Strobl/Austria, 21. November

Thomas, David A., Robin J. Ely, (1996): Making Dif-

ferences Matter: A New Paradigm for Managing

Diversity; in: HBR 74, 79-90

Ass.-Prof. Dr. Cäcilia Innreiter-Moser

Johannes Kepler Universität Linz

Institut für Organisation und Institut für Frauen- und

Geschlechterforschung

Altenberger Straße 69

A-4040 Linz, Österreich

Dr. Harald Stummer

Johannes Kepler Universität Linz

Institut für Internationale Unternehmensführung und

Institut für Personalwirtschaft

Altenberger Straße 69

A-4040 Linz, Österreich 